\title{
Towards a Wearable Augmented Reality Visor for High-Precision Manual Tasks
}

\author{
$1^{\text {st }}$ Nadia Cattari* \\ ${ }^{1}$ Department of Translational Research \\ and New Technologies in Medicine \\ and Surgery \\ ${ }^{2}$ EndoCAS Center \\ University of Pisa \\ Pisa, Italy \\ 0000-0002-7091-2908
}

\author{
$1^{\text {st }}$ Roberta Piazza* \\ ${ }^{3}$ Information Engineering Department \\ ${ }^{2}$ EndoCAS Center \\ University of Pisa \\ Pisa, Italy \\ 0000-0003-4116-8312
}

\author{
$3^{\text {rd }}$ Renzo D'Amato \\ ${ }^{3}$ Information Engineering Department \\ ${ }^{2}$ EndoCAS Center \\ University of Pisa \\ Pisa, Italy \\ 0000-0003-2477-8813
}

\author{
$5^{\text {th }}$ Marina Carbone \\ ${ }^{3}$ Information Engineering Department \\ ${ }^{2}$ EndoCAS Center \\ University of Pisa \\ Pisa, Italy \\ 0000-0002-1004-6964 \\ $8^{\text {th }}$ Vincenzo Ferrari \\ ${ }^{3}$ Information Engineering Department \\ ${ }^{2}$ EndoCAS Center \\ University of Pisa \\ Pisa, Italy \\ 0000-0001-9294-2828
}

\author{
$6^{\text {th }}$ Sara Condino \\ ${ }^{3}$ Information Engineering Department \\ ${ }^{2}$ EndoCAS Center \\ University of Pisa \\ Pisa, Italy \\ 0000-0001-5235-0414
}

\author{
${ }^{3}$ Information Engineering Department \\ ${ }^{2}$ EndoCAS Center \\ University of Pisa \\ Pisa, Italy \\ 0000-0001-6773-3741
}

\begin{abstract}
Augmented Reality (AR) systems based on headmounted displays intrinsically provide the user with an egocentric and natural viewpoint, and for this reason they are deemed as the most efficient solution for those tasks manually performed under user's direct vision. They are commonly classified according to the AR paradigm implemented: Video See-Through (VST) and Optical See-Through (OST). A current research goal for these systems is to determine either of which of the two modalities is to be considered the best solution for the guidance of high-precision manual tasks. To answer this question, in this paper we report the outcomes of two user studies performed by our research team that involved a commercially available OST (Microsoft HoloLens) and a customized new-concept VST head-mounted display. The average error in tracing a line under AR aid is considerably inferior in VST user study than the one performed with OST device $(1 \mathrm{~mm}$ and $5.8 \mathrm{~mm}$, respectively). However, some of the advantages offered by OST device are not negligible, thus a hybrid solution implementing the two paradigms can overcome the limitations of both technologies.
\end{abstract}

Index Terms-augmented reality, head-mounted display, opti-

* Nadia Cattari and Roberta Piazza contributed equally to this paper (email: nadia.cattari@endocas.unipi.it; roberta.piazza@endocas.unipi.it).

This work was supported by the HORIZON2020 Project VOSTARS, Project ID: 731974. Call: ICT-29-2016 Photonics KET 2016. The research leading to these results has received partial funding from the Italian Ministry of Education and Research (MIUR) in the framework of the CrossLab project (Departments of Excellence) of the University of Pisa, laboratory of Augmented Reality. cal see-through, video see-through, high-precision manual task.

\section{INTRODUCTION}

Augmented Reality (AR) systems allow the real-time visualization of additional information within the real environment for an enhanced user interactive experience [1]. AR technology has been employed for the development of wearable visors, more commonly referred to as Head-Mounted Displays (HMDs), whose uses range in many application fields, from gaming [2] to industry [3], engineering design [4] to medicine [5], and derived combinations [6]. The increasing interest in these new devices is due to the possibility of carrying out freehand movements under the user's direct vision maintaining his/her egocentric perspective [7], [8].

The enhancement of the real world view with the superimposition of computer-generated three-dimensional (3D) objects can be obtained either with an Optical See-Through (OST) or a Video See-Through (VST) HMDs [9]. In both cases, visors embody semi-transparent displays placed in front of the user's eyes; the distinction between the two approaches consists in the see-through paradigm they adopt. Whereas with OST the user's direct view of the real scene is preserved and only the virtual content is projected onto the display [10], with 
VST visors the real scene, acquired from one or two external cameras, is shown on the display together with the virtual enrichment only after it has been consistently merged with it [11].

The use of commercial AR system based on HMD technology is investigated by many authors in several contexts [12]. One of the research areas impacted by the use of AR HMD is the image-guided surgery, potentially providing surgeons with a useful planning and/or navigation tool that integrates medical imaging into surgical workflow [13]-[16]. However, existing perceptual issues are related to the rendering appropriate focus cues [17], [18], especially during manual task: the interaction between real and virtual objects, which are projected at a fixed focal distance (generally between $2 \mathrm{~m}$ and infinity), causes perceptual conflicts, such as vergenceaccommodation conflict (VAC) and focus rivalry (FR) [19], [20] due to the incapability of human eye to keep in focus both the virtual and real content simultaneously. In addition, commercial AR HMD have been developed as general-purpose products: the absence of a framework specifically dedicated to medical applications, a platform-dependent tracking method and/or a complex calibration technique represent other major drawbacks [21].

Nowadays, OST HMDs are considered for surgical applications since they provide a full-scale resolution of the real scenario and can allow the safely completion of the intervention even if the display fails, since the direct view of reality is not compromised [22]. On the other hand, the VST paradigm can offer an accurate registration of virtual content to the real scene at the cost of camera-mediated view. This can be translated into a higher system lag (from $50 \mathrm{~ms}$ to $80 \mathrm{~ms}$ ), due to the time needed for the processing of the images grabbed by the cameras, the tracking, and the visualization of contents [23]. However, this can be exploited as an advantage as the acquired video of the real scene can be delayed to match the virtual elements. In OST HMDs, system lag affects only the virtual content, resulting in spatial and temporal misalignment between the real and virtual information that can negatively interfere with the user performance and/or cause discomfort. Despite the development of increasingly sophisticated OST headsets, perceptual and technological limitations have to be addressed, as they preclude the employment of such devices to accurately guide and perform surgical manual tasks where a high level of precision is demanded (i.e. incision/cutting of human body tissues). By means of example, the intrinsic virtual-to-real latency of the OST is one of these limitations. To overcome the latency purely due to the tracking process, hybrid tracking strategies, such as the one integrated in the Microsoft HoloLens, that combine the results of optical selftracking with the speed of inertial measurement units (IMUs) are required.

To provide a reasonable answer to which of the two seethrough paradigms is the most effective tool in guiding highprecision manual tasks, in this work we describe two recent user studies carried out by our research group employing an advanced OST and a custom-made VST HMDs, respectively.

\section{High-Precision Manual Task with AR HMDs}

In the following subsections, two user studies for the evaluation of AR headsets employed as guidance for twodimensional (2D) high-precision manual tasks are reported (Fig.1). In particular, the first work concerns one of the most advanced HMD OST on the market used for exploring the perceptual conflicts effects in AR-guided "connect-thedots" tasks [24]. The second study was designed to evaluate the accuracy when tracing a simulated incision line with a customized VST visor [25].
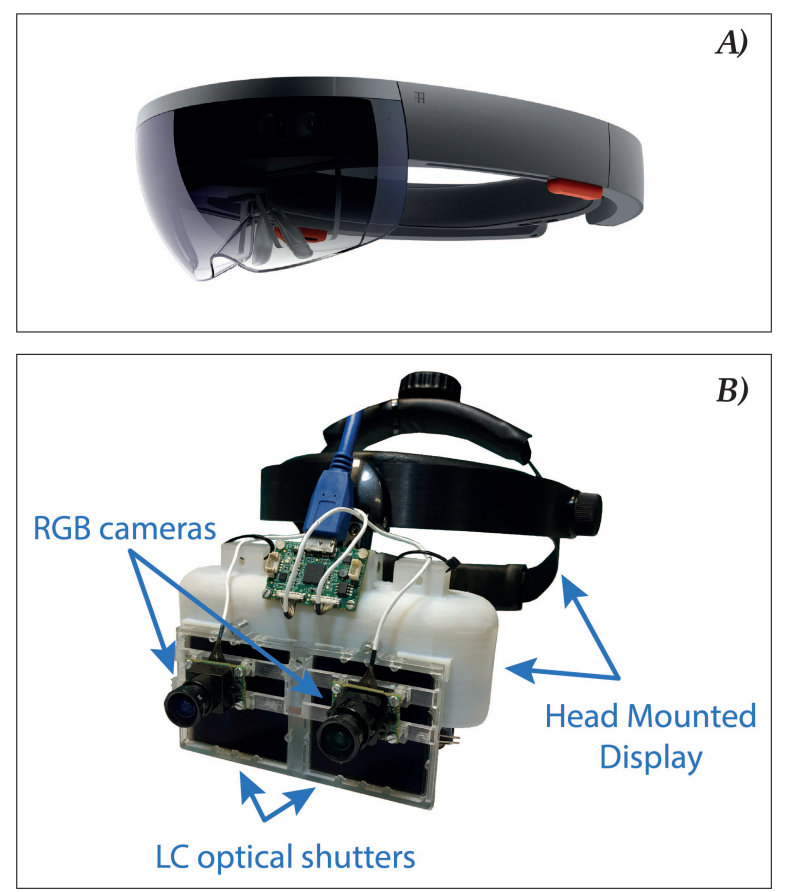

Fig. 1. AR headsets employed for the execution of high-precision manual task: the OST Microsoft Hololens (A), and a modified HDM (B).

In both studies, a number of participants from technical employees and university students were recruited. Moreover, the methods employed to perform test are reported, together with the qualitative and quantitative evaluation results.

\section{A. Study A: commercial OST device}

In order to investigate the effect of VAC and FR, together and separately, during the execution of AR guided manual task with a commercial OST headset, an user study has been first conducted by Condino et al. [24].

As shown in Fig.2, the experimental study involved the use of the Microsoft HoloLens to conduct a simple task such as drawing consecutive lines (14 in total) on a paper, using a pen and a ruler, following a series of points marked with numbers. Twelve sequences of numbered dots were created and randomly associated for each participant (20 in total) with the following four test modalities: monocular and binocular naked-eye $\left(N E_{m o n}\right.$ and $\left.N E_{\text {bin }}\right)$, and monocular and binocular AR guidance $\left(A R_{\text {mon }}\right.$ and $\left.A R_{\text {bin }}\right)$. More specifically, in the NE modalities, the numbered points series were printed on a paper and the subject was asked to draw lines on an overlaying 
tracing sheet. As for the AR-guided session, the binocular test was planned to determine the effect of the FR and VAC together, while the monocular version aimed to evaluate the effect the FR solely. Moreover, in AR modalities, user's eye was obliged to focus on the virtual numbered points and real objects (pen, ruler, and paper). Each participant was asked to accomplish three times the "connect-the-dots" tasks in each modalities.
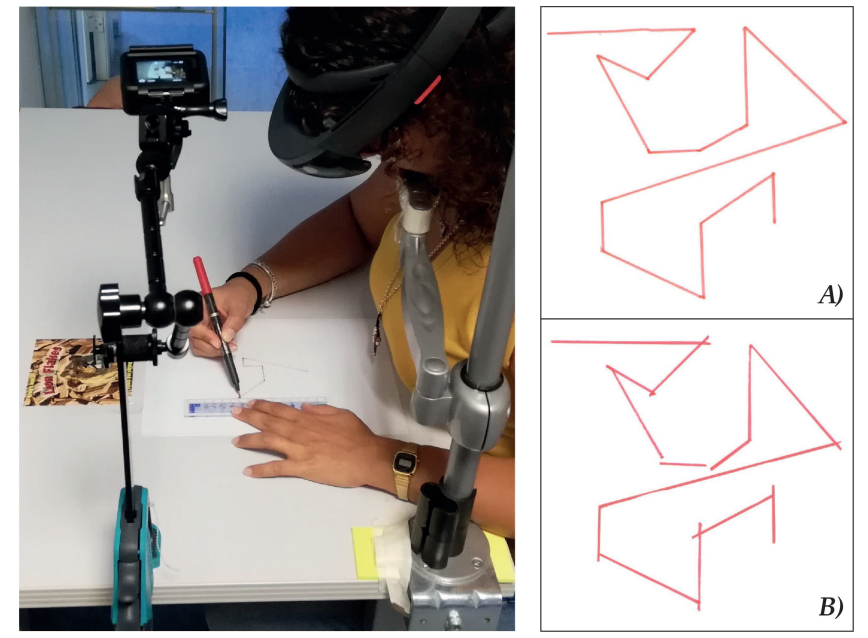

Fig. 2. OST HMD Study: on the left, the experimental set up during a testing session; on the right, examples of one task respectively performed with both naked-eye, i.e. without AR (A), and with AR (B) modalities.

Each trial was analyzed to evaluate the accuracy in connecting dots. More in particular, for each testing session, the maximum and mean difference in length between each line drawn by the participant and the correspondent virtual segment, and the maximum and mean gap between the endpoints End $_{i}$ and Start $_{j}$ of each pair $(i, j)$ of consecutive lines were calculated. Time to complete tests was also recorded.

All testing sessions were successfully accomplished by all the participants who did not indicate any appreciable spatial shift of the virtual information. The maximum error committed by participants in AR modality is $5.9 \pm 1.7 \mathrm{~mm}$ in length versus $2.8 \pm 1.2 \mathrm{~mm}$ in $\mathrm{NE}$ modality, with gaps up to $5.8 \mathrm{~mm}$ and $2.3 \mathrm{~mm}$, respectively.

Collected data (difference in length and gap between traced lines and predicted ones) were subjected to a post hoc analysis with Wilcoxon signed-rank tests via Bonferroni correction to examine whether the modality test has an effect on the subject performance. A statistically significant difference, set at $p<0.012$, depending on test modality, was observed between the two monocular tests $\left(N E_{\text {mon }} v s\right.$. $\left.A R_{\text {mon }}\right)$ and between the two binocular tests $\left(N E_{\text {bin }} v s . A R_{b i n}\right)$. However, no significant differences are shown between monocular and binocular tests neither for naked-eye $\left(N E_{\text {mon }} v s . N E_{b i n}\right)$ nor for AR-guided modalities $\left(A R_{\text {mon }}\right.$ vs. $\left.A R_{\text {bin }}\right)$.

Difference in perceived workload according to both different AR modalities $\left(A R_{\text {mon }}\right.$ and $\left.A R_{\text {bin }}\right)$ and user characteristics (experience with AR, with Hololens and with virtual reality), and subjective evaluation of focus cues were also examined by administering questionnaires to subjects (NASA-TLX and Likert). In the Likert questionnaire, participants declared to have perceived virtual content (dots and numbers) as clear and sharp in AR-guided visualization modes, but they expressed a neutral opinion regarding: the ability to contemporaneously focus at the virtual and real objects, the perception of visual discomfort due to blur, the comfort of using the AR guidance for the selected task, the trustability of the AR modality to successfully guide manual task, their confidence in the precision they can reach in manual tasks guided by the AR modality. Finally, users stated to have perceived more visual fatigue during binocular than monocular AR tests, but this difference was not statistically significant. In both comparisons, statistical analysis did not identify significant differences neither between the monocular and binocular AR modalities (Wilcoxon Signed-Rank test) nor between groups with different experience with augmented and virtual reality systems (Kruskal-Wallis test).

\section{B. Study B: customized VST device}

As aforementioned, VST paradigm allows to obtain a more accurate registration between the real scene and the virtual enrichment, even if provided with a higher latency time. As described in our previous works [26], [27], a commercial binocular OST visor can be re-engineered to obtain a HMD that could yield both the video and optical see-through paradigms. As a matter of fact, assembling together a HMD, two RGB cameras and a pair of liquid-crystal (LC) optical shutters, they obtained a headset able to switch from OST to VST mode, and viceversa, thanks to the electronically transparency change of these latter.

Recently, Cutolo et al. [25] presented a software framework for the deployment of AR application for the in-situ visualization of medical imaging data. This highly configurable and computationally efficient platform was employed with the custom-made HMD in an AR application designed to aid a high-precision manual task. In particular, they designed an experimental user study to evaluate quantitatively and qualitatively the reliability of the new concept headset coupled with the software platform in guiding in VST mode a highprecision manual task, i.e. a digital simulated tissue incision.

Twelve participants were asked to wear the customized VST HMD and to perform the same simulated incision task under two conditions: with naked-eye and with AR guidance. A tablet notebook was used as digital surface of incision and a digital pen as a digital scalpel. Each participants was asked to trace ten different spline lines (five open curves and five closed curves) with the digital stylus on the display of the tablet under both conditions.

The quantitative analysis was performed by measuring the similarity between the virtual spline associated to the planned line of incision and the actual curve traced by the subject, both with and without the AR guidance. The authors used the Hausdorff distance to determine the "closeness" between these two trajectories. Moreover, the time for completing the manual task under both conditions was also measured. 

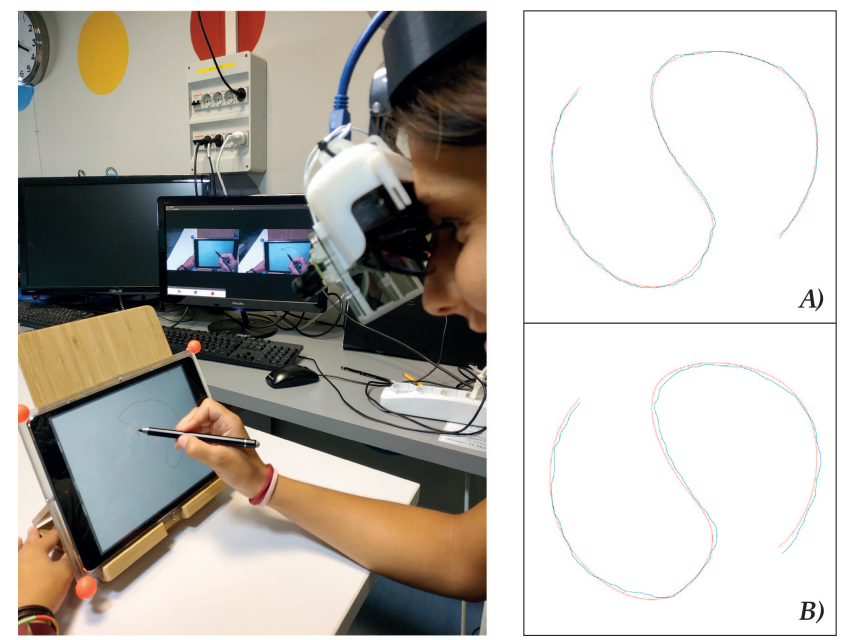

Fig. 3. VST HMD Study: on the left, the experimental set up during a testing session; on the right, examples of one task respectively performed with both naked-eye (A), and with AR (B) modalities. Note the line of incision drawn (in blu) following the planned one (in red).

The quantitative analysis showed positive results: the average difference between the planned and the traced spline line was less than $1 \mathrm{~mm}$ for the AR test. It is worth mentioning that, on average, the subjects performed mildly better with the naked eye rather than with the HMD (only $0.07 \mathrm{~mm}$ of spatial difference). On the other hand, the completion times were generally higher for the naked-eye than for the the tests under the AR guidance.

A post hoc analysis with Wilcoxon signed-rank test on both the Hausdorff distance and the time of completion was carried out to establish whether the users performance depends on the guidance modality. Results showed a statistically significant difference $(p<0.05)$ in the accuracy of the performance with the naked eye compared with the AR guidance, while, as for the completion time, the statistically significant difference was in favour of the AR guidance. Moreover, to determine if the performance is influenced by the subject previous experience with AR, HMD or digital pen, the Mann-Whitney U test was conducted, revealing no significant differences $(p>0.05)$. Similarly, no statistically significant differences in completion times were found in users who have experience with AR and with HMD, whereas for the users with some experience with the digital pen a statistically significant difference was detected.

Data for the qualitative analysis were gathered after completing both groups of tests; each subject was asked to fill in a Likert questionnaire in terms of usability, functionality and technological acceptance. The qualitative results show an overall positive opinion over the user study, especially regarding the ease-to-use and for the comfort of the task. The level of frustration experienced by the participants during the test were generally low.

\section{DISCUSSION AND CONCLUSION}

Recent studies raised the evidence that HMDs are emerging as the most ergonomic and efficient solution for the guidance of manual tasks, for their ability to leave unaltered the egocentric viewpoint of the user. However, currently issue regards which of the two paradigms available, optical and video see-through, is the most suitable for supporting highprecision manual tasks. To this end, in this work we reported two user studies carried out by our research team involving a commercially available OST and a customized VST.

The results of the two studies are summarized in Table I.

TABLE I

SUMMARY TABLE OF THE TWO USER STUDIES.

\begin{tabular}{|c|c|c|c|c|}
\hline & \multicolumn{2}{|c|}{ Study A } & \multicolumn{2}{c|}{ Study B } \\
\hline $\begin{array}{c}\text { See-Through } \\
\text { Paradigm }\end{array}$ & \multicolumn{2}{|c|}{ OST } & \multicolumn{2}{c|}{ VST } \\
\hline Device & \multicolumn{2}{|c|}{$\begin{array}{c}\text { Microsoft } \\
\text { Hololens }\end{array}$} & \multicolumn{2}{c|}{$\begin{array}{c}\text { Customized } \\
\text { HMD }\end{array}$} \\
\hline $\begin{array}{c}\text { Number } \\
\text { of participants }\end{array}$ & \multicolumn{2}{|c|}{20} & \multicolumn{2}{|c|}{12} \\
\hline $\begin{array}{c}\text { Error } \\
\text { Evaluation }\end{array}$ & \multicolumn{2}{|c|}{$\begin{array}{c}\text { Planned/drawn lines } \\
\text { length difference }\end{array}$} & $\begin{array}{c}\text { Planned/drawn lines } \\
\text { Hausdorff distance }\end{array}$ \\
\hline $\begin{array}{c}\text { Test } \\
\text { Modalities }\end{array}$ & NE & AR & NE & AR \\
\hline $\begin{array}{c}\text { Number } \\
\text { of trials }\end{array}$ & 6 & 6 & 10 & 10 \\
\hline $\begin{array}{c}\text { Quantitative } \\
\text { Results [mm] (std) }\end{array}$ & $\begin{array}{c}2.8 \\
(1.2)\end{array}$ & $\begin{array}{c}5.9 \\
(1.7)\end{array}$ & $\begin{array}{c}0.91 \\
(0.14)\end{array}$ & $\begin{array}{c}0.98 \\
(0.17)\end{array}$ \\
\hline $\begin{array}{c}\text { Qualitative } \\
\text { Analysis }\end{array}$ & \multicolumn{2}{|c|}{ Neutral } & \multicolumn{2}{c|}{ Positive } \\
\hline
\end{tabular}

In literature, there is no standardization of reported inaccuracies for image-guided surgery (IGS) systems [28]. In fact, all errors are integrative and may be be classified depending on their source (imaging-related, registration-related, humanrelated) [29], or based on the standard classification between fiducial localization error, fiducial registration error, and target registration error. Considering that an image-guided system for complex manual task in surgery is recommended when the accuracy is less than 1-2 $\mathrm{mm}$ [28], [30], the outcomes of the experiments with HoloLens suggested that this device is not recommendable for aiding high-precision procedures in the peripersonal space (equal to or less than arm length) [24]. Moreover, the average error made by the participants was considerable and significantly higher than the one made during the execution of the task without the AR guidance. In addition, the general opinion of the users about the visor was tentative: they were not able to say whether it was reliable or not, since they could not predict the accuracy with which they performed the requested task.

On the contrary, the results of the VST experiments were very encouraging. Though the subjects performed slightly better in the naked-eye tests, the AR experiments still showed promising results, with a very low discrepancy between the planned and the drawn line. Furthermore, the comments on the comfort, usability and reliability of the visor were generally 
positive. On this basis, we can state that in case of manual tasks which require a high level of precision the VST system is to be preferred, since the new-concept AR HMD coupled with the software platform is a reliable solution.

The major limitation of the present work is that it was not possible a direct comparison between the two systems since the user study protocols, in particular referred to the experimental set up and the requested tasks (drawing a line in a 2D space), were different. Planned future work should include the design of a common protocol for testing properly and in an unbiased way the two paradigms for the guidance of 2D tracing tasks. In addition, a further comparison study should be addressed to confirm the efficiency of the AR platform for guiding high-precision also in a 3D space, e.g. following the protocol we have recently reported in [31], where the customized VST mechanism features a photon-to-photon perceived latency of at least $67 \mathrm{~ms}$.

It is worth considering that, although the VST device offers better performances, it features drawbacks that cannot be ignored. For example, if a malfunctioning occurs, the direct vision of the operating field is not preserved, thus preventing the user to safely proceed with the task completion. This issue is easily avoided in the OST system which instead features an unhindered view of the real scene. Therefore, combining the advantages of both see-through paradigms can potentially offer the best solution for an AR guiding system capable to adapt to different conditions. This aspect has already been addressed within European project VOSTARS (Video and Optical See-Through Augmented Reality Surgical Systems [32]). Indeed, the software platform and the the new-concept headset described in [17] and proposed by the aforementioned project, are designed for the deployment of both VST and OST AR application. This would allow our group to perform a common test in both modalities by simply switching from one to the other using the same device.

\section{ACKNOWLEDGMENT}

This work was supported by the European Project VOSTARS-H2020 Call ICT-29-2016 G.A. (731974). The authors would like to thank Umberto Fontana, Stefano Mascioli and $\mathrm{PhD}$ Cinzia Freschi for providing their support in this project.

\section{REFERENCES}

[1] R. T. Azuma, "A Survey of Augmented Reality," Presence Teleoperators Virtual Environ., vol. 6, pp. 355-385, August 1997.

[2] P. Das, M. Zhu, L. McLaughlin, Z. Bilgrami and R.L Milanaik, "Augmented Reality Video Games: New Possibilities and Implications for Children and Adolescents," Multimodal Technologies Interact., vol. 1, April 2017.

[3] P. Fraga-Lamas, T. M. FernáNdez-CaraméS, Ó. Blanco-Novoa and M. A. Vilar-Montesinos, "A Review on Industrial Augmented Reality Systems for the Industry 4.0 Shipyard," IEEE Access, vol. 6, pp. 13358-13375, February 2018.

[4] X. Wang, S.K. Ong, and A.Y.C. Nee, "A comprehensive survey of augmented reality assembly research," Adv. Manuf., vol. 4, pp. 1--22, January 2016.

[5] M. Eckert, J.S. Volmerg, and C.M. Friedrich, "Augmented Reality in Medicine: Systematic and Bibliographic Review," JMIR Mhealth Uhealth, vol. 7, April 2019.
[6] R.M. Viglialoro, S. Condino, G. Turini, M. Carbone, V. Ferrari, and M. Gesi, "Review of the Augmented Reality Systems for Shoulder Rehabilitation," Information, vol. 10, p. 154, April 2019.

[7] P. Vávra, J. Roman, P. Zonča, P. Ihnát, M. Němec, J. Kumar, N. Habib, and A. El-Gendi "Recent Development of Augmented Reality in Surgery: A Review," J Healthc Eng., vol. 2017, 4574172, August 2017.

[8] N. Cattari, F. Cutolo, R. D'amato, U. Fontana and V. Ferrari, "Toedin vs Parallel Displays in Video See-Through Head-Mounted Displays for Close-Up View," IEEE Access, vol. 7, pp. 159698-159711, October 2019.

[9] J. P. Rolland and H. Fuchs, "Optical versus video see-through meadmounted displays in medical visualization," Presence Teleoperators Virtual Environ., vol. 9, pp. 287-309, June 2000.

[10] S. A. Benton, "Selected Papers on Three-Dimensional Displays," SPIE Opt. Eng. Press. San Diego, May 2001.

[11] N. S. Holliman, N. A. Dodgson, G. E. Favalora, and L. Pockett, "Threedimensional displays: A review and applications analysis," IEEE Trans. Broadcast., vol. 57, pp. 362-371, June 2011.

[12] G.F. Trotta, S.Mazzola, G. Gelardi, A. Brunetti, N. Marino, and V. Bevilacqua "Reconstruction, Optimization and Quality Check of Microsoft HoloLens-Acquired 3D Point Clouds," Neural Approaches to Dynamics of Signal Exchanges. Smart Innovation, vol 151, pp. 98-93, September 2019.

[13] T. M. Peters, "Image-guidance for surgical procedures," Phys Med Biol., vol. 51, pp. R505-R540, June 2006.

[14] T. Sielhorst, M. Feuerstein, and N. Navab, "Advanced Medical Displays: A Literature Review of Augmented Reality," J. Disp. Technol., vol. 4, pp. 451--467, December 2008.

[15] F. Cutolo, M. Carbone, P. D. Parchi, V. Ferrari, M. Lisanti, and M. Ferrari, "Application of a new wearable augmented reality video seethrough display to aid percutaneous procedures in spine surgery," Lect. Notes Comput. Sc., vol. 9769, pp. 43-54, June 2016.

[16] A. Pepe, G.F. Trotta, P. Mohr-Ziak, C. Gsaxner, J. Wallner, V. Bevilacqua and J. Egger "A Marker-Less Registration Approach for Mixed Reality-Aided Maxillofacial Surgery: a Pilot Evaluation,” J Digit Imaging, vol. 32, pp. 1008-1018, September 2019.

[17] F. Cutolo, "'Letter to the editor on "augmented reality based navigation for computer assisted hip resurfacing: A proof of concept study"," Ann. Biomed. Eng., vol. 47, pp. 2151-2153, June 2019.

[18] M. Carbone, R. Piazza, and S. Condino, "Commercially Available Head-Mounted Displays Are Unsuitable for Augmented Reality Surgical Guidance: A Call for Focused Research for Surgical Applications," Surg. Innov., February 2019.

[19] K. Oshima, K. R. Moser, D. C. Rompapas, J. E. Swan, S. Ikeda, G. Yamamoto, T. Taketomi, C. Sandor, and H. Kato, "SharpView: Improved clarity of defocussed content on optical see-through headmounted displays," P Ieee Virt Real Ann, pp. 253-254, March 2016.

[20] H. Hua, "Enabling focus cues in head-mounted displays," Proc. IEEE, vol. 105, pp. 805--824, May 2017.

[21] B. Marques, F. Roy, N. Haouchine, E. Jeanvoine, S. Cotin, R. Plantefeve, and I.Peterlik, "Framework for augmented reality in Minimally Invasive laparoscopic surgery," Proc. 17th Int. Conf. E-health Networking, Application Services (HealthCom), pp. 22-27, October 2015.

[22] L. Qian, A. Barthel, A. Johnson, G. Osgood, P. Kazanzides, N. Navab, and B. Fuerst, "Comparison of optical see-through head-mounted displays for surgical interventions with object-anchored 2D-display," Int J Comput Assist Radiol Surg, vol. 12, pp. 901-910, March 2017.

[23] J.P. Freiwald, N. Katzakis, and F. Steinicke, "Camera Time Warp: Compensating Latency in Video See-Through Head-Mounted-Displays for Reduced Cybersickness Effects," Proc. 24th ACM Symposium on Virtual Reality Software and Technology, pp. 1-7, November 2019.

[24] S. Condino, M. Carbone, R. Piazza, M. Ferrari, and V. Ferrari, "Perceptual Limits of Optical See-Through Visors for Augmented Reality Guidance of Manual Tasks," IEEE Trans Biomed Eng., vol 67, pp. 411419, May 2019.

[25] F. Cutolo, B. Fida, N. Cattari, and V. Ferrari, "Software Framework for Customized Augmented Reality Headsets in Medicine," IEEE Access, vol. 8, pp. 706-720, December 2019.

[26] F. Cutolo, U. Fontana, and V. Ferrari, "Perspective preserving solution for quasi-orthoscopic video see-through HMDs," Technologies, vol. 6, pp. 1-20, March 2018.

[27] U. Fontana, F. Cutolo, N. Cattari, and V. Ferrari, "Closed - Loop Calibration for Optical See-Through Near Eye Display with Infinity 
Focus," Proc. IEEE Int. Symp. Mixed and Augmented Reality (ISMAR), pp. 51-56, October 2018

[28] R.F. Labadie, B.M. Davis, and J.M. Fitzpatrick, "Image-guided surgery: what is the accuracy?," Curr Opin Otolaryngol Head Neck Surg, vol. 13, pp. 27-31, February 2005.

[29] G. Widmann, R. Stoffner, R. Bale, "Errors and error management in image-guided craniomaxillofacial surgery," Oral Surg Oral Med Oral Pathol Oral Radiol Endod., vol. 107, pp. 701-715, May 2009.

[30] R. Hussain, A. Lalande, C. Guigou and A. Bozorg Grayeli, "Contribution of Augmented Reality to Minimally Invasive Computer-Assisted Cranial Base Surgery," IEEE J Biomed Health Inform, November 2019.

[31] S. Condino, B. Fida, M. Carbone, L. Cercenelli, G. Badiali, V. Ferrari and F.Cutolo, "Wearable Augmented Reality Platform for Aiding Complex 3D Trajectory Tracing," Sensors, vol. 20, p. 1612, March 2020.

[32] VOSTARS H2020 project, G.A. 731974. 